\title{
Formation of Morphologically Unusual Features, Associated with Immunodeficiencies, in Lymph Nodes of Gnotobiotic Rats Exposed to a Conventional Milieu*
}

\author{
G. Sainte-Marie and F.-S. Peng \\ Département d'Anatomie, Université de Montréal, Montréal, Québec, Canada
}

Received May 11, 1989

\begin{abstract}
Summary. Athymic animals are characterized by unusual features in lymph nodes, which are indicative of immunodeficiencies. These features include hypertrophy of follicles, atrophy of the peripheral cortex above the center of deep cortex units, and the formation of lymphocyte clusters at the periphery of these units as well as of compartment replicas in the capsules. Such features were recently observed in some nodes of a minority of aged euthymic animals and we concluded that they probably also reflected immunodeficiencies, since immunodeficiencies may emerge in aging euthymic animals. In an attempt to validate this conclusion, we exposed one-year-old gnotobiotic animals to a conventional milieu, thereby presumably rendering these euthymic animals somewhat immunodeficient, and checked their nodes for unusual features. Nodal unusual features, similar to those encountered in nodes of athymic and of some aged euthymic animals, arose rapidly and the ex-gnotobiotic animals either manifested signs of infections or died. These findings support our previous conclusion that the arising of such features reflects a progressive emergence of a certain state of immunodeficiency with aging.
\end{abstract}

The lymph nodes of athymic nude animals have peculiar unusual features which reflect their state of immunodeficiency. These features are particularly well developed in the much antigen-challenged cervical and mesenteric nodes, as well as in the older nude animals (SAINTE-MARIE et al., 1984b). We recently observed similar unusual features in some nodes of a minority of aged euthymic animals (SAINTE-MARIE and PENG, 1987a), where they were less developed than in corresponding nodes of nude animals. We suggested that these unusual features probably also reflected a certain state of immunodeficiency, which may emerge in aging euthymic animals (HANNA et al., 1967; NoRDIN and MAKINODAN, 1974; SHIGEMOTO et al., 1975; MAKINODAN et al., 1977). In another respect, it has been shown that germ-free rats develop severe diarrhea or die within about a week following exposure to a conventional milieu (GUSTAFSSON, 1967). This condition was attributed to infection by microorganisms of the open milieu and to the incapacity of such animals, which have a poorly developed lymphoid system (THORBECKE, 1959; BAUER, 1968; WOSTMAN et al., 1970), to cope with the challenge. Infection is, indeed, the most obvious manifestation of inadequate immune responses, i.e., of a state of immunodeficiency taken in the broad sense (HAYWARD, 1977).

In an attempt to validate our proposal that the formation of concerned unusual features in nodes of aging animals reflects antigenic challenges following the emergence of a state of immunodeficiency, we checked in the present study as to whether or not such unusual features might occur in nodes of oneyear-old gnotobiotic euthymic animals, presumably rendered somewhat immunodeficient by exposure to a conventinal environment.

\section{MATERIALS AND METHOD}

Twelve gnotobiotic Sprague-Dawley rats of both sexes were obtained from the Research Institute of the National Research Council (Ottawa, Ontario) where they had served as breeders. Cultures had been

*This work was funded by the Medical Research Council of Canada. 
carried out every two weeks in the laboratory of origin to ascertain the gnotobiotic state of the animals. Six animals were sacrified as controls. The others were placed and maintained in a conventional environment for two months at 12 months of age, and treated in the standard manner. Animals were sacrificed with chloroform and cervical, mesenteric, brachial, axillary, inguinal and popliteal nodes were removed from each animal. The nodes were fixed in a solution of Bouin-Hollande for $48 \mathrm{~h}$ and paraffinembedded. Each node was cut serially at $7 \mu \mathrm{m}$; one out of every 15 sections was mounted and stained with the technique of Dominici.

\section{RESULTS AND DISCUSSION}

\section{Nodes of control gnotobiotic animals}

Recent studies of ours reexamined the histology of the rat lymph node, that of its deep cortex in particular. It was shown that the node is morphologically and functionally divided into "compartments" and that the deep cortex is constituted of "deep cortex units", each compartment having such a unit
(BÉlisle and SAinte-Marie, 1981). Figure 1 schematizes a compartment of the rat node, and thus defines the used terms.

The nodes of the control gnotobiotic animals had usual structures as reported previously (THORBECKE, 1959; WOSTMAN and GORDON, 1960; GuSTAFSSON, 1967). In almost all nodes, however, some elements of these structures were absent or poorly developed which is characteristic of germ-free animals. Hence, the extrafollicular zone situated directly above medulla was much lymphocyte-depleted and had generally poorly developed high endothelial venules (BÉLISLE et al., 1982), the follicles lacked a nodule or germinal center (BAuER, 1968; Wostman et al., 1970) and the medullary cords had very few plasmacytes (THOR. BECKE, 1959; Gustafsson, 1967; POllard, 1967). This absence of nodules and of plasmacytes reflects a very low level of humoral immune responses (BAUER, 1968; WOSTMAN et al., 1970) and shows that almost all nodes of these animals were very little, if at all, challenged by antigens provoking humoral immune responses. In the same nodes, the centers of the deep cortex units, which are the sites of the nodal events of cellular immune responses (OORT and TURK, 1965), unusually exhibited an homogeneous aspect and did

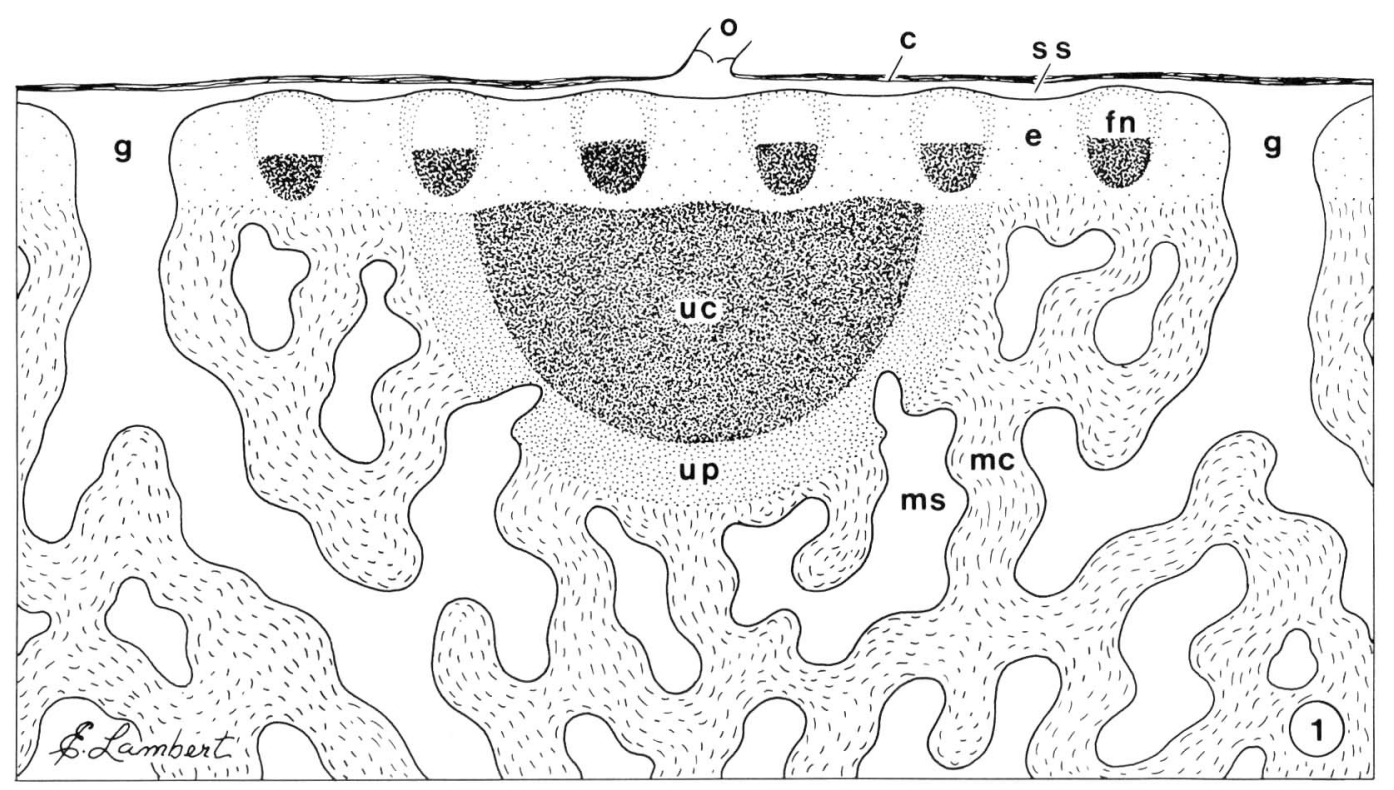

Fig. 1. Diagram of a "compartment" of the rat lymph node, centered on the related afferent lymphatic opening $(o)$ which abuts in the subcapsular sinus (ss) across the capsule $(c)$. The corresponding portions of subcapsular sinus and of peripheral cortex are delimited, on either side, by a gap $(g)$ where the subcapsular sinus connects directly with medullary sinuses. The peripheral cortex has an extrafollicular zone $(e)$ spacing folliculo-nodules $(f n)$, i.e., follicles with a nodule or germinal center. A compartment also includes a "deep cortex unit", comprising a center $(u c)$ and a periphery $(u p)$, as well as medullary sinuses ( $m s)$ and cords $(m c)$. 
Fig. 2. Cervical node of a twelve-monthold gnotobiotic rat. Because of the homogeneous aspect of the lymphoid parenchyma, the different lymphoid structures are difficult to identify. The dark dots are mast cells present in medullary sinuses. $\times 35$

Fig. 3. Cervical node of a fourteen-monthold ex-gnotobiotic rat, exposed to a conventional environment for two months. Note the heterogeneous aspect of the lymphoid parenchyma. Very large folliculo-nodule $(f)$ occurs above the center of the deep cortex unit $(u)$. Dark "lymphocyte clusters" (arrows) formed at the periphery of the deep cortex unit. Plasmacytic medullary cords are wide. $\times 25$

Fig. 4. Mesenteric node of the same animal as in Fig. 3. The node has hypertrophied, dark, and protruding folliculonodules $(f)$, as well as lymphocyte clusters (arrows). $\times 25$

Fig. 5. Caecal node of the same animal as in Fig. 3. The node has hypertrophied folliculo-nodules $(f)$. A compartment "replica" overlies the pale subcapsular sinus (arrowheads) of the node. The arrow points to a nodule (germinal center) present in a follicle of the replica. $\times 35$
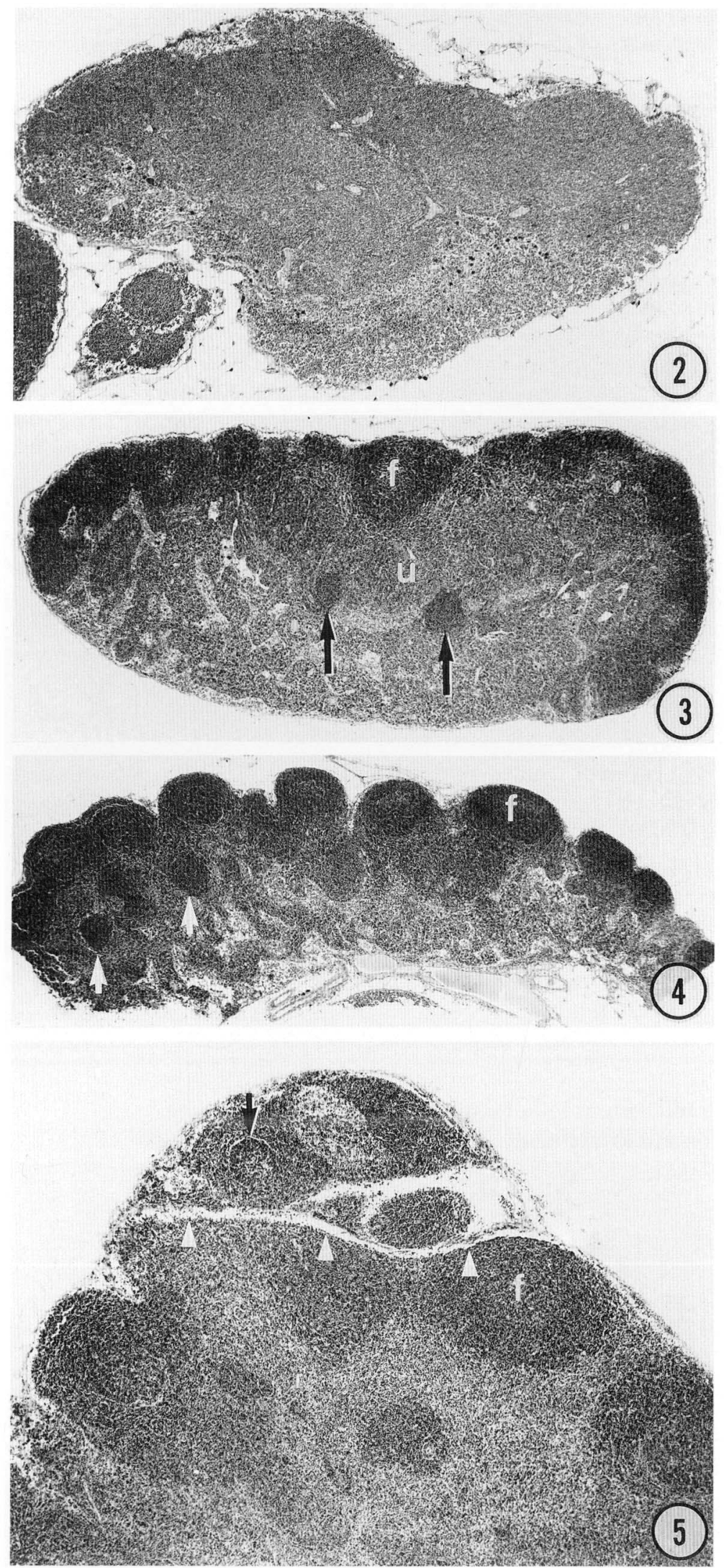
not contain blasts. Such an homogeneous aspect of the centers of the deep cortex units appears to reflect the absence, or the almost complete absence, of challenging antigens provoking cellular immune responses (BÉLISLE et al., 1982). Hence, the whole lymphoid parenchyma appeared almost uniform (Fig. 2), differing markedly from its appearance in nodes of standard animals. Indeed, cellular density and staining intensity of the lymphoid parenchyma were quite uniform throughout the nodes of these gnotobiotic animals, so that the different nodal structures could be distinguished only with difficulty, unlike the situation in standard animals. This uniform aspect of the lymphoid parenchyma was due to the fact that it was almost exclusively populated by small lymphocytes as previously observed in germ-free animals (BAUER et al., 1963), because of a lack of antigenic stimuli and an absence of immune responses. This contention is supported by the finding that the lymphoid parenchyma of stimulated nodes from gnotobiotic animals exposed to a conventional environment was not uniform in appearance (see below).

Exceptionally, some compartments of a few cervical and mesenteric nodes contained variably sized nodules (germinal centers) and numerous plasmacytes. The stimulation of these compartments may have resulted from a site-restricted invasion of the drained tissues, possibly by alimentary proteins. However, the presence of very small numbers of plasmacytes in most of the other nodes indicated a very weak antigenic stimulation, or an entry of plasmacytic precursors formed elsewhere (FORD and GOWANS, 1969; VAN DER BRUGGE-GAMELKOORN et al., 1987). Lastly, the nodes of the control gnotobiotic rats did not exhibit the unusual features which occur in nodes of athymic animals.

\section{Nodes of gnotobiotic animals exposed to a conven- tional environment}

Within two weeks of exposure to a conventional environment, two of the animals died with a severe diarrhea. Since infection is the most obvious manifestation of defective immune responses (HAYWARD, 1977), the rapid death of these animals apparently resulted from their incapacity to cope with a sudden invasion of micro-organisms, i.e., from the rapid onset of a severe state of immunodeficiency. The remaining animals became sick with inflamed eyes and snouts, but did not die. The resistance of germfree animals to infection is influenced by age (OUT . ZEN, 1969), the nature of the infecting agents (WOST . MAN and GORDON, 1960; GUSTAFSSON, 1967; OUTZEN,
1969), the manner in which animals are contaminated (Wostman and GoRdon, 1960; Gustafsson, 1967), and individual features. For instance, germ-free mice, infected with Salmonella typhimurium, die within two weeks when less that 60 -day-old, but about within one week when more than 180-day-old (OUTZEN, 1969). On the other hand, after infection by enema of a suspension of conventional intestinal flora, 90-day-old germfree rats remain healthy (WOSTMAN and GoRDON, 1960). But when germ-free rats are contaminated randomly by being placed in a conventional milieu, the animals develop severe diarrhea and one out of three die within about a week (GUSTAFSSON, 1967), just as we observed here. Death and disease were attributed to infection by micro-organisms present in the open milieu (GUST AFSSON, 1967). Hence, our ex-gnotobiotic rats probably died or became sick because they were exposed to a septic milieu and were relatively aged. The poorly developed lymphoid organs of their immune system apparently could not cope with some infecting micro-organisms, which is what the term immunodefeciency expresses here in line with the concept that immunodeficiency has a "spectrum of severity" (OUTZEN, 1969). The occurrence of relatively milder forms of immunodeficiency in most of our ex-gnotobiotic animals, as compared to those who died rapidly, can account for their greater longevity. The question remains as to whether or not such animals would eventually have recovered, if allowed to live longer.

The overall appearance of the nodes of our exgnotobiotic animals was comparable to that of corresponding nodes of normal animals. High endothelial venules were often well developed, variably-sized nodules (germinal centers) occurred in follicles, plasmacytes often abounded in medullary cords, and the centers of deep cortex units generally had a heterogeneous aspect. The presence of lymphocytes in the unit centers of the nodes of the ex-gnotobiotic animals, although some cellular immune responses were probably defective, may be explained by the fact that they, contrary to athymic animals, were not devoid of T-cells. The development of these normal features reflected ongoing immune responses against challenging antigens. However, all ex-gnotobiotic animals had nodes with some morphologically unusual features characteristic of nodes of nude animals, but which were less developed than in nude animals. These features were particularly evident in cervical and mesenteric nodes. Normal animals exposed to the same conventional environment developed no such unusual features. Therefore, the emergence of these features in the much antigen-challenged cervical and 
mesenteric nodes of our ex-gnotobiotic animals, indicated that the immune system of these animals had not yet coped, or could not cope fully with all the challenging antigens or micro-organisms, i.e., that they were partially immunodeficient. This can account for the development of some unusual features in their nodes, together with the greater development of the usual features of the nodes. This situation bear some ressemblance to that observed in nodes of incompletely neonatally thymectomized animals or in nodes of thymus-grafted nude animals (SAINTE-MARIE et al., 1984a, 1986). Under these various conditions, unusual features are fewer or less developed than in nude animals because of many successful immune responses.

HA YWARD (1977) pointed out that immunodeficiencies can be due to various causes, ranging from the lack of a primary lymphoid organ or lymphoid cell line to malnutrition. Since the thymuses of our 12-month-old gnotobiotic rats were generally smaller than those of young rats, inadequate immune responses may have resulted, to a certain degree, from thymic involution. However, the emergence of a state of immunodeficiency in these animals was probably primarily due to the overriding effect of certain micro-organisms in the draining nodes rather than to a lack of proper lymphocytes.

The observed unusual features included: 1) a light to moderate atrophy of the peripheral cortex overlying the centers of deep cortex units, 2) hypertrophy of "follicles", mainly those present above the peripheries of deep cortex units, and 3) the formation of "lymphocyte clusters" at the periphery of deep cortex units (Figs. 3, 4). The significance of these unusual features, particularly with respect to either humoral or cellular immune responses, was discussed elsewhere (SAINTE-MARIE and PENG, 1983; SAINTE-MARIE et al., 1984c). In a few nodes, we noted an additional unusual feature recently described in nodes of nude animals which consisted in the accumulation of very dark, much elongated and often pyknotic lymphocytes in areas of a node's subcapsular sinus (SAINTEMARIE and PENG, 1990).

We also observed "lymphocyte infiltration" of the capsule in many nodes, as well as the presence of small to medium-sized "compartment replicas" in cervical and caecal nodes. Lymphocyte infiltration of a capsule is an early stage in the formation of a compartment replica. A replica represents a partial duplication of a somewhat deficient node compartment, occurring in a mirror-image and on the outer wall of the subcapsular sinus (SAINTE-MARIE and PENG, 1987b). It was interpreted as a compensatory process. Compartment replicas were found so far in nodes of aging athymic rats only, their tardy formation apparently accompanying a pronounced state of immunodeficiency. Hence, the formation of compartment replicas in nodes of the present animals would indicate the emergence of a rather severe state of immunodeficiency, as was also indicated by the rapid death of some of these animals and the very ill state of the others. We observed compartment replicas in the caecal nodes but not in the other mesenteric nodes, even though their capsules were infiltrated by lymphocytes. This emphasizes again that the degree of development of nodal features, or the occurrence of peculiar activities, varies with anatomical sites (SAINTE-MARIE and PENG, 1983, 1987a). Basic differences were noted between replicas of our ex-gnotobiotic animals and those of athymic animals. Firstly, a nodule (germinal center) occurred in follicles of the replicas of the ex-gnotobiotic animals (Fig. 5), unlike in athymic animals. These nodules could form, because the ex-gnotobiotic animals were not devoid of T-cells which are essential for nodule genesis (JACOBSON et al., 1974; KoT ANI et al., 1974). Secondly, the replicated node compartments of our ex-gnotobiotic animals were only slightly atrophied in comparison to those of athymic animals. This probably reflects successful immune responses occurring against part of the challenging antigens or micro-organisms since, again, these ex-gnotobiotic animals were not devoid of T-cells. Furthermore, this explanation would also account for the smaller size of replicas in the ex-gnotobiotic than in the athymic rats.

One might expect us to prove, by immunological tests, that the nodal unusual features affecting the ex-gnotobiotic animals resulted from a deficiency related to one or several antigens inducing cellular and/or humoral immune responses. Unfortunately, such a proof would be quite difficult to obtain, for diverse reasons. For instance, to establish a direct link between nodal anomalies and immunodeficiencies, one would have to identify, and test an animal's response to, the numerous challenging antigens or micro-organisms. The testing of antigens other than those actually challenging an animal would be unconclusive. Moreover, it would also be necessary to prove that unresponded antigens are actually challenging those node(s) or node compartment(s) exhibiting anomalies. This would require a very large number of animals and nodes, and would represent an overwhelming task. Instead, we are seeking more simple ways to establish links between a state of immunodeficiency and nodal unusual features. Nature has fortunately provided us with nude ani- 
mals and it has been established that they are immunodeficient and that their nodes develop predictable unusual features not encountered in young normal animals. It is therefore logical to conclude that, in nude animals, the unusual features affecting nodal structures involved in humoral immune responses reflect deficiencies of $\mathrm{T}$-dependent humoral responses, and likewise for cellular immune responses. It is also reasonable to conclude that the same unusual features found in some other animals affected, or presumably affected, by immunodeficiency are most probably due to deficiencies of either type of immune responses. We had so far encountered such unusual features in thymus-grafted nude animals and in partially neonatally thymectomized animals (SAINTEMARIE et al., 1984a, 1986), as well as in a minority of aged euthymic animals (SAINTE-MARIE and PENG, 1987a). Our present observations add to this list and strenghten our argument for a causal relationship between such nodal unusual features and a state of immunodeficiency. All the above animals were probably affected by variable forms of immunodeficiency, but more mildly than nude animals. Accordingly, their nodal unusual features were relatively less developed than in nude athymic animals.

In summary, we observed, in one-year-old gnotobiotic euthymic animals exposed to a conventional environment and apparently severely infected, rapid formation of many morphologically unusual features which are common in nodes of immunodeficient nude animals. This supports our previous suggestion that the presence of such unusual features in nodes of some aging euthymic animals, probably reflects immunodeficiencies which emerge with age (HANNA et al., 1967; NORDIN and MAKINODAN, 1974; SHIGEMOTO et al., 1975).

Acknowledgments. The authors thank Dr. J. L. BHASIN of The Research Institute of The National Council (Ottawa, Ontario) for having kindly provided us the gnotobiotic animals and Miss G. GUAY for her technical assistance.

\section{REFERENCES}

BAUER, H.: Defence mechanisms in germ-free animals: II. Cellular defence mechanisms. In: (ed. by) M. E. COATES: The germ-free animal in research. Academic Press, New-York, 1968 (p. 210-226).
Bauer, H., R. E. Horowitz, S. M. Levenson and H. PAPPER: The response of the lymphatic tissue to the microbial flora. Studies on germ-free mice. Amer. J. Pathol. 42: 471-483 (1963).

BÉlisle, C. and G. SaInte-MARIE: Tridimensional study of the deep cortex of the rat lymph node. III. Morphology of the deep cortex units. Anat. Rec. 199: 213-226 (1981).

Bêlisle, C., G. Sainte-Marie and F. S. Peng: Tridimensional study of the deep cortex of the rat lymph node. VI. The deep cortex units of the germ-free rat. Amer. J. Pathol. 107: 70-78 (1982).

Brugge-Gamelkoorn, G. J., N. VAN der Kors and N. VAN ROOIJEN: Development of specific antibody-forming cells in various lymphoid organs of rabbit after intravenous antigen administration. Anat. Rec. 217: 56-60 (1987).

Ford, W. L. and J. L. Gowans: The traffic of lymphocytes. Semin. Hematol. 6: 67-76 (1969).

GoRdon, H. A. and B. S. Wostmann: Morphological studies on the germ-free albino rats. Anat. Rec. 137:6570 (1960).

Gustafsson, B. E.: Introduction of specific microorganisms into germ-free animals. In: (ed. by) G. E. W. WOLSTENHOLME and M. O'CONNOR: Nutrition and infection (Ciba Foundation study group 31). JA Churchill, London, 1967 (p. 3-17).

Hanna, M. G., P. Nettesheim, L. Ogden and T. MakinoDAN : Reduced immune potentiallity of aged mice: significance of morphologic changes in lymphatic tissue. Proc. Soc. Exp. Biol. Med. 125: 882-886 (1967).

HaYwARD, A. R.: Immunodeficiency. Edward Arnold, London, 1977.

Jacobson, E. B., L. H. CAPorale and G. J. Thorbecke: Effect of thymus cell injections on germinal center formation in lymphoid tissues of nude (thymusless) mice. Cell. Immunol. 13: 416-430 (1974).

Kotani, M., Y. Nawa, H. Fuji, T. Fukumoto, M. MiYamoto and A. Yamashita: Involvement of thymus cells in the formation of germinal centers. Acta Anat. 90: 585-590 (1974).

Makinodan, T., M. L. Heidrick and A. A. Nordin: Immunodeficiency and autoimmunity in aging. In: (ed. by) D. BERGSMA: Immunodeficiency in man and animals. Krieger Publishing, New York, 1977 (p. 193-201).

Nordin, A. A. and T. Makinodan: Humoral immunity in aging. Fed. Proc. 33: 2033-2035 (1974).

OorT, J. and J. L. TURK: A histological and autoradiographic study of lymph nodes during the development of contact sensitivity in the guinea pig. Brit. J. Exp. Pathol. 46: 147-154 (1965).

OUTZEN, H. C.: Aging and resistance to infection in germ-free C3Hf mice. In: (ed. by) E. A. MirAND and N. BACK: Germ-free biology. Experimental and clinical aspects. Plenum Press, New York, 1969 (p. 207-217).

Pollard, M.: Germinal centers in germ-free animals. In: (ed. by) H. CotTIER, N. OdARTChEnko, R. ShindLER and C. C. ConGDON: Germinal centers in immune responses. 
Springler-Verlag, New York, 1967 (p. 343-346).

Sainte-Marie, G. and F. S. Peng: Structural and cell population changes in the lymph nodes of the athymic nude mouse. Lab. Invest. 49: 420-429 (1983).

: Morphological anomalies associat ed with immunodeficiencies in the lymph nodes of aging mice. Lab. Invest. 54: 598-610 (1987a).

- : The formation of "compartment replicas" in the lymph nodes of aging athymic nude rats. Cell Tiss. Res. 248: 323-333 (1987b).

: Atrophy of lymph node compartments related to an entry of altered lymphocytes under conditions of immunodeficiency. Cell Tiss. Res. (1990, in press).

Sainte-Marie, G., M. Pelletier and F. S. Peng: Modifications of the structures of the rat lymph nodes by neonatal thymectomy. Thymus 6: 309-323 (1984a).

Sainte-Marie, G., F. S. Peng and M. Pelletier: Development of the lymph nodes in the very young, and their evolution in the mature, nude rat. Devel. Comp. Immunol. 8: 695-710 (1984b).

Sainte-Marie, G., F. S. Peng and D. Pritchard: Tridimensional study of the deep cortex of the rat lymph node. VIII. The deep cortex unit of athymic nude rats. Anat. Rec. 209: 95-104 (1984c).

Sainte-Marie, G., M. Pelletier and F. S. Peng: Morphological anomalies in the lymph nodes of 13-monthold thymus-grafted nude mice. Thymus 8: 77-90 (1986).
Shigemoto, S., S. Kishimoto and Y. Yamamura: Changes of cell-mediated cytotoxicity with aging. J. Immunol. 115: 307-309 (1975).

Thorbecke, G. J.: Some histological and functional aspects of lymphoid tissue in germ-free animals. Ann. New York Acad. Sci. 78: 237-246 (1959).

Wostman, B. S. and H. E. GoRdon: Electrophoretic studies on the serum protein pattern of the germ-free rat and its changes upon exposure to a conventional bacterial flora. J. Immunol. 84: 27-31 (1960).

Wostman, B. S., J. R. Pleasants, P. Bealmear and P. W. KINCADE: Serum proteins and lymphoid tissues in germ-free mice fed a chemically defined, water soluble, low-molecular weight diet. Immunology 19: 443-448 (1970).
Dr. Guy SAINTE-MARIE

Département d'Anatomie Université de Montréal

C. P. 6128, Succ. A

Montréal (Québec)

Canada H3C 3J7 